\title{
Root cause analysis of pseudohyperkalemia in a patient with focal segmental glomerulosclerosis: A case report
}

\author{
Sriharsha Talluri*1, Satish C Talluri ${ }^{2}$ \\ ${ }^{1}$ Geisinger Commonwealth School of Medicine, Scranton, United States \\ ${ }^{2}$ OSS Hospital, York, United States
}

Received: July 3, 2017

Accepted: September 18, 2017 Online Published: September 21, 2017

DOI: $10.5430 /$ crim.v $4 \mathrm{n} 4 \mathrm{p} 21$

URL: https://doi.org/10.5430/crim.v4n4p21

\begin{abstract}
Pseudohyperkalemia, also known as factitious or spurious hyperkalemia, is predominantly due to hemolysis during the collection process of the specimen. Pseudohyperkalemia is a condition in which the falsely elevated potassium can cause significant clinical dilemma as patients may be given unnecessary and harmful treatment for hyperkalemia. In addition, pseudohyperkalemia can also mask hypokalemia resulting in patients potentially not receiving adequate potassium replacement. Thus, it is pivotal for clinicians and laboratory personnel to have a high index of suspicion for this condition in order for the patient to receive appropriate treatment. We present a case of pseudohyperkalemia without obvious hemolysis due to the use of a 23-gauge needle blood draw in a patient with stage IV chronic kidney disease from focal segmental glomerulosclerosis.
\end{abstract}

Key Words: Pseudohyperkalemia, Chronic kidney disease, Hemolysis, 23-gauge needle

\section{INTRODUCTION}

Critical hyperkalemia is a medical emergency in which immediate treatment has to be initiated in order to prevent hemodynamic, neurological, and respiratory consequences in addition to sudden death due to malignant arrhythmias. ${ }^{[1]}$ Red blood cell lysis causes leakage of potassium into the plasma causing pseudohyperkalemia. However, this error is usually recognized and reported by the laboratory personnel to the clinical team. There are several other mechanisms wherein potassium could be falsely elevated, from preanalytical causes and analytical errors to medical conditions. An effective history of present illness, past medical history, medication record, physical examination, EKG, prior lab work if available, and more importantly, awareness of this condition, can help the clinical team in administering the right treatment to the patient. Hyperkalemia symptoms are usually nonspe- cific and patients can present with nausea, vomiting, dyspnea, tachypnea, chest pain, palpitations, or even flaccidity. EKG changes in hyperkalemia include tented $\mathrm{T}$ waves, short P-R intervals, and widened QRS complexes. ${ }^{[2]}$ Urgent treatment is necessary in patients with EKG changes in order to bring decrease the potassium level and prevent fatal arrhythmias. A quick root cause analysis should be undertaken in collaboration with laboratory personnel when pseudohyperkalemia is suspected.

\section{Case presentation}

A 53-year-old male with a past medical history significant for stage IV chronic kidney disease secondary to focal segmental glomerulosclerosis was admitted to our specialty orthopedic elective hospital for left medial knee replacement. His preoperative creatinine was 2.5 , GFR $26 \mathrm{ml} / \mathrm{min}$, and serum

\footnotetext{
*Correspondence: Sriharsha Talluri; Email: stalluri@tcmc.edu; Address: Geisinger Commonwealth School of Medicine, 831 Adams Ave., Apt \#19, Scranton, PA 18510, United States.
} 
potassium was 5.4 (normal up to 5.1). His other preoperative laboratory results were within normal limits including complete blood count. The patient follows up with his nephrologist every 3 months, which included a visit prior to his surgery. He also had a preoperative medical evaluation performed by an internist. The patient was on lisinopril for his hypertension and kidney disease. His other significant past medical history included hypertension, hyperlipidemia, gout, and sleep apnea. His current medications included lisinopril, allopurinol, and rosuvastatin. The patient was instructed to drink three bottles of Gatorade (each bottle has $75 \mathrm{mg}$ of potassium for a total of $225 \mathrm{mg}$ ) as part of preoperative hydration protocol of the hospital, and he also received 2 liters of ringer lactate during his surgery. He was not given any medications during the perioperative period that would cause hyperkalemia.

A repeat potassium level was done on the patient postoperatively shortly after coming to the surgical floor since his preoperative potassium was elevated. Laboratory personnel immediately notified the clinical team that the serum potassium came back at a critical level of 6.8 and also informed the team that the sample was not hemolyzed. The patient denied any symptoms, was placed on telemetry, and an EKG was performed which did not show any changes of hyperkalemia. Complete blood count did not reveal any leukocytosis or thrombocytosis and creatinine remained stable at 2.6. The same sample was retested at our hospital and also run through a point of care analyzer, which confirmed the initial hyperkalemia of 6.8. Since we are an elective hospital without ICU facilities, the patient was transferred to the nearby academic hospital (10 minutes away) with ICU capabilities by ACLS ambulance for management of the patient's critical hyperkalemia. Prior to his transfer, the patient's clinical course was discussed with his nephrologist who works at the academic hospital. Pseudohyperkalemia was entertained in the differential diagnosis, but treatment was deferred until seen by his nephrologist. Repeat potassium level done at the academic hospital showed a normal potassium level of 4.8 , and the patient was sent back to our hospital the same evening. The potassium was repeated again the next morning at our hospital and the level came back again as critical at 6.8 (no hemolysis was identified), and this was confirmed again by retesting and through point of care analyzer. His creatinine level remained stable at 2.6. No immediate treatment was given as the patient remained asymptomatic and EKG did not reveal any changes of hyperkalemia.

Pseudohyperkalemia due to lab collection error or processing error was entertained immediately and a quick root cause analysis was performed looking into the causes of pseudohyperkalemia in a systematic manner. It was ascertained that the phlebotomist was using a 23-gauge butterfly needle for blood draw that caused the patient's pseudohyperkalemia. Laboratory personnel did not detect hemolysis on visual inspection. A repeat sample was immediately processed using a 22-gauge straight needle, which revealed a normal potassium level of 4.9. The patient was explained in detail about his pseudohyperkalemia and was discharged home the same day in stable condition. Our patient had all the ingredients to develop critical hyperkalemia including elevated baseline potassium, underlying stage IV chronic kidney disease, ACE inhibitor therapy, Gatorade ingestion, and Ringer lactate administration. Only a high index of suspicion on the part of clinical team averted any unnecessary treatment for the patient.

\section{Discussion}

Potassium is one of the most commonly ordered laboratory test. ${ }^{[3]}$ Pseudohyperkalemia is a condition wherein potassium levels are falsely elevated due to various errors in the pre-analytical phase of blood collection, analytical phase, or due to underlying medical conditions. It has been reported that preanalytical errors account for majority of pseudohyperkalemia and analytical errors follow in second. ${ }^{[4]}$ There are several mechanisms for pseudohyperkalemia in which hemolysis is the most common cause. Hemolysis could result from multiple factors, including but not limited to, excessive application of suction, use of small gauge needles, vigorous mixing, increased turbulence, and tourniquet application time. Delay in processing, contaminations of specimen, or incorrect centrifugation are some other analytical factors that can cause pseudohyperkalemia. ${ }^{[5]}$

Analytical errors are less common causes of hyperkalemia since laboratories usually use standardized analyzers and the equipment is monitored and assessed frequently. Some errors also occur during the reporting of the results as well as due to fist clenching and not following the order of draw. ${ }^{[5]} \mathrm{A}$ complete blood count usually gives a clue to the diagnosis of pseudohyperkalemia if leukocytosis or thrombocytosis are present. However, for other causes a high index of suspicion is necessary to diagnose the condition, especially if hemolysis is not evident. Pseudohyperkalemia, in our case, was due to non-detection of hemolysis since laboratory personnel used visual inspection instead of standard analyzers, which are more accurate in detecting hemolysis. ${ }^{[5]}$ There are several medical conditions that can cause pseudohyperkalemia and the most common ones are diseases causing thrombocytosis or leukocytosis. ${ }^{[1]}$ Leukemias, lymphomas and myeloproliferative disorders are all implicated in causing pseudohyperkalemia without hemolysis. Pseudohyperkalemia occurring in thrombocytosis has no linear correlation to the platelet 
count. ${ }^{[6]}$

We wanted to highlight the importance of having a high index of suspicion or awareness of this condition as there are several factors which can lead to pseudohyperkalemia, even in the absence of hemolysis, as reported in our case. A quick root cause analysis should be performed in conjunction with laboratory personnel to identify the factors leading to pseudohyperkalemia and appropriate measures should be taken in order to avoid any unnecessary and dangerous treatment to the patient. ${ }^{[7]}$ Clinicians should be aware of the possibility of pseudohyperkalemia masking true hypokalemia, depriving the patient of required potassium replacement. Clinicians should also be familiar with how hemolysis is detected in their own laboratories, especially in smaller hospitals. Whether or not laboratory personnel report hemolysis via visual interpretation of the specimen, which is not as reliable as detection by automated analyzers, is an important distinction to be aware of. Detection through automation should become the standard of care in all facilities. ${ }^{[8]}$ Our hospital, after a thorough review of the root cause analysis of pseudohyperkalemia, has begun to implement measures to prevent this critical laboratory error. We are in the process of updating our equipment to automated analyzers.

In conclusion, our patient's pseudohyperkalemia was due to use of 23-gauge needle, which caused hemolysis but was not detected. This was because our laboratory personnel used visual inspection process to detect hemolysis, which is an unreliable method. We propose that clinicians work in close conjunction with laboratory personnel to arrive to a conclusion quickly for the cause for pseudohyperkalemia. These patients may receive inappropriate treatment, and for the same reason patients may not receive adequate treatment if pseudohyperkalemia is masking true hypokalemia.

\section{Conflicts OF InTEREST Disclosure}

The authors have declared no conflicts of interest.

\section{REFERENCES}

[1] Gibbs MA, Tayal VS. Electrolyte Disturbances. In: Marx JA, Hockberger RS, Walls RM, editors. Rosen's Emergency Medicine: Concepts and Clinical Practice. Seventh Edition. New York, NY: Mosby; 2009. p. 1620-2.

[2] Wrenn KD, Slovis CM, Slovis BS. The ability of physicians to predict hyperkalemia from the ECG. Ann Emerg Med. 1991; 20: 1229-32. https://doi.org/10.1016/S0196-0644(05)81476-3

[3] Forsman RW. Why is the laboratory an afterthought for managed care organizations? Clin Chem. 1996; 42: 813-6. PMid:8653920

[4] Stankovic AK, Smith S. Elevated serum potassium values: The role of preanalytic variables. Am J Clin Pathol. 2004; 121: S105-12. https://doi.org/10.1309/UEPQUM11WH9P8JNY

[5] Asirvatham JR, Moses V, Bjornson L. Errors in Potassium Measurement: A Laboratory Perspective for the Clinician. North American
Journal of Medical Sciences. 2013; 5(4): 255-9. PMid:23724399

https://doi.org/10.4103/1947-2714.110426

[6] Glick MR, Ryder KW, Glick SJ, et al. Unreliable visual estimation of the incidence and amount of turbidity, hemolysis, and icterus in serum from hospitalized patients. Clin Chem. 1989; 35: 837-9. PMid:2720981

[7] Sevastos N, Theodossiades G, Efstathiou S, et al. Pseudohyperkalemia in serum: The phenomenon and its clinical magnitude. J Lab Clin Med. 2006; 147: 139-44. PMid:16503244 https: //doi.org/10.1016/j.lab.2005.11.008

[8] Lippi G, Luca SG, Blanckaert N, et al. Multicenter evaluation of the hemolysis index in automated clinical chemistry systems. Clin Chem Lab Med. 2009; 47: 934-9. PMid:19548845 https: //doi.org/10.1515/CCLM.2009.218 